\title{
TUNE SHIFTS AND SPREADS DUE TO SHORT AND LONG RANGE BEAM-BEAM INTERACTION
}

\author{
L.F. WANG*, C. ZHANG, S.H. WANG and Z.Y. GUO \\ Institute of High Energy Physics Chinese Academy of Sciences, Beijing, P. R. China
}

\section{Abstract}

The performance of existing and proposed collider is often limited by the beam-beam interaction. The tune shift and spread from head-on and long-range beam-beam interactions for both round and flat Gaussain beam have been studied with analysis method in this paper. These formulas are useful for multi-bunch colliders where both short-range and long-range beam-beam interactions should be considered.

\section{INTRODUCTION}

Beam-beam effects are one of the most important phenomena which limits the luminosity in a storage ring. Luminosity is proportional to the average current and the vertical tune shift. The vertical beam-beam tune shift is limited to a value around $0.03 \sim 0.05$, so the main thrust of our effects is to increase the average beam current by adding more bunches. The long-range beam-beam effects put a limitation on the bunch number in many case such as in CESR, LEP and B-Factory. The shift and spread from head-on and long range beam-beam interactions for round beam have been studied in many papers [1, 2]. Analysis of the shift and spread for general bunch shape is presented in this paper.

\section{HEAD-ON AND LONG RANGE TUNE SHIFT}

The most general electron beam charge distribution, which will be considered, is a Gaussian distribution in three dimensions, given by

$$
\rho=\frac{e N}{(2 \pi)^{3 / 2} \sigma_{x} \sigma_{y} \sigma_{z}} \exp \left[-\frac{1}{2}\left(\frac{x^{2}}{\sigma_{x}^{2}}+\frac{y^{2}}{\sigma_{y}^{2}}+\frac{z^{2}}{\sigma_{z}^{2}}\right)\right],
$$

Here, $e$ is electron charge. The co-ordinate axes are labelled $x$ for the radially outwards direction, $y$ for the direction perpendicular to the median plane, and $z$ along the equilibrium orbit.

The long-range beam-beam tune shift for two bunches with separation $X$ and $Y$ can be expressed as

$$
\xi_{x}=\frac{N \beta_{x} r_{e}}{2 \pi \gamma} \int_{0}^{\infty}\left(1-\frac{2 X^{2}}{2 \sigma_{x}^{2}+t}\right) \frac{\exp \left[-\frac{X^{2}}{2 \sigma_{x}^{2}+t}-\frac{Y^{2}}{2 \sigma_{y}^{2}+t}\right]}{\left(2 \sigma_{x}^{2}+t\right)^{3 / 2}\left(2 \sigma_{y}^{2}+t\right)^{1 / 2}} d t,
$$

$$
\xi_{y}=\frac{N \beta_{y} r_{e}}{2 \pi \gamma} \int_{0}^{\infty}\left(1-\frac{2 Y^{2}}{2 \sigma_{y}^{2}+t}\right) \frac{\exp \left[-\frac{X^{2}}{2 \sigma_{x}^{2}+t}-\frac{Y^{2}}{2 \sigma_{y}^{2}+t}\right]}{\left(2 \sigma_{x}^{2}+t\right)^{1 / 2}\left(2 \sigma_{y}^{2}+t\right)^{3 / 2}} d t,
$$

where, $\beta_{x, y}$ is the betatron function at the encounter point, $r_{e}=e^{2} /\left(4 \pi \varepsilon_{0} m_{0} c^{2}\right)$ is the classical particle radius and $\gamma=\left(1-v^{2} / c^{2}\right)^{-1 / 2}$ is the Lorentz factor.

When beam-beam collisions are head-on, which means $X$ and $Y$ are both zero in the above formulae, equations 2 and 3 give the well known formulae for the beam-beam parameters

$$
\begin{aligned}
& \xi_{x 0}=\frac{N \beta_{x} r_{e}}{2 \pi \gamma \sigma_{x}\left(\sigma_{x}+\sigma_{y}\right)}, \\
& \xi_{y 0}=\frac{N \beta_{y} r_{e}}{2 \pi \gamma \sigma_{y}\left(\sigma_{x}+\sigma_{y}\right)} .
\end{aligned}
$$

Figures 1-2 show the relative beam-beam parameters Vs beam transverse separation for a flat beam with $\sigma_{x}=0.645952 \mathrm{~mm}$ and $\sigma_{y}=0.02691 \mathrm{~mm}$. The horizontal beam-beam parameter is negative when the horizontal beam separation is larger than $1.32 \sigma_{x}$, which means the beam-beam force defocus the particle in the horizontal direction. However, the beam-beam kicks are always focus force when the two beams are separated in only vertical plane. Comparing fig.(1) with fig. (2), we can see the horizontal required beam relative separation is smaller than the vertical ones for flat bunch $\left(\sigma_{x}>>\sigma_{y}\right)$. Therefore, we usually chose horizontal separation other than vertical separation. Figures 3-4 show the relative beam-beam parameter change with transverse separation for round beam.

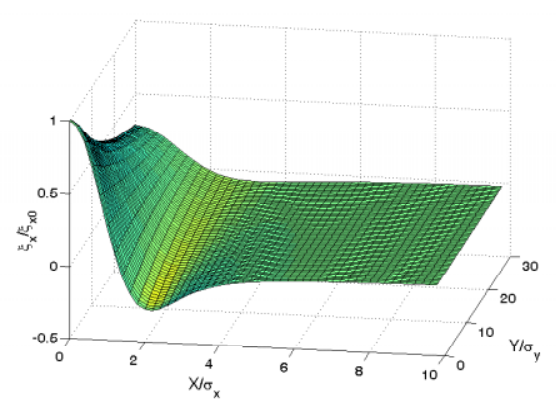

Figure 1 Horizontal beam-beam parameters Vs beam horizontal and vertical separation for flat bunch

\footnotetext{
*Corresponding author, High Energy Accelerator Research organization, KEK Accelerator Laboratory, 1-1 Oho, tsukuba-shi, Ibaraki 305-0801, Japan.wanglf@post.kek.jp
} 


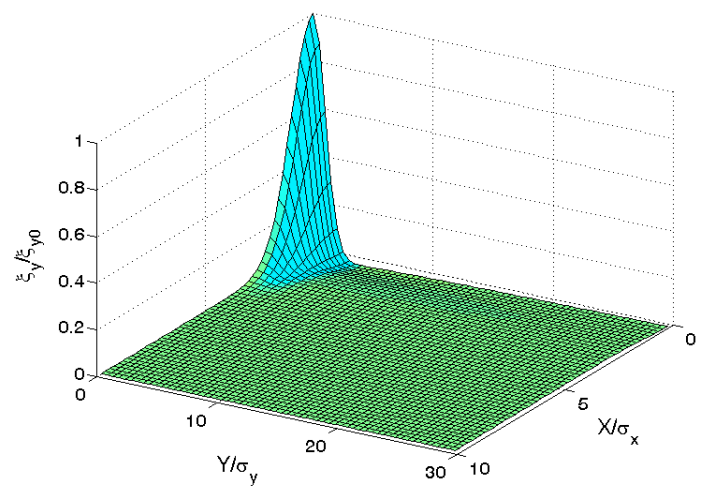

Figure 2: Vertical beam-beam parameters Vs beam horizontal and vertical separation for flat bunch

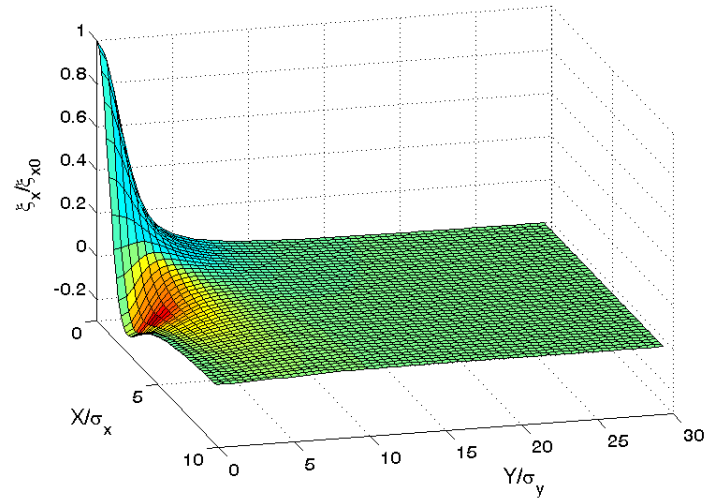

Figure 3: Horizontal beam-beam parameters Vs beam separation for round bunches $\left(\sigma_{y}=\sigma_{x}\right)$

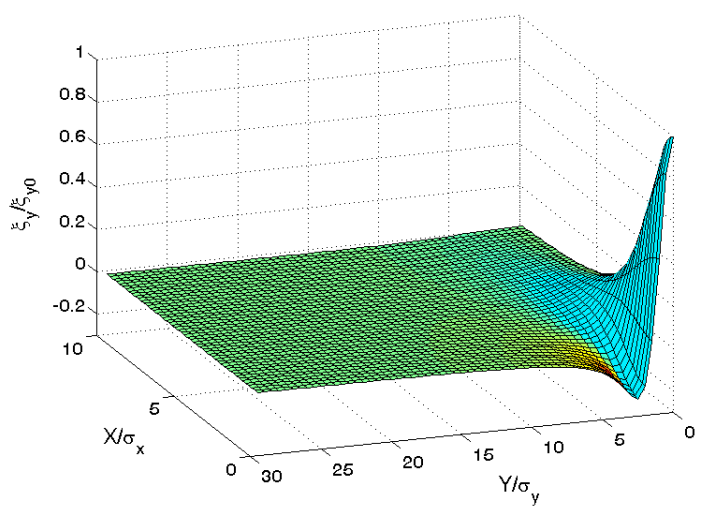

Figure 4: Vertical beam-beam parameters Vs beam separation for round bunches $\left(\sigma_{y}=\sigma_{x}\right)$

When bunches are separated in only one direction, such as CESR and BEPCII where two beams are separated in horizontal direction, one can get the approximate long range beam-beam parameter expression in the case of large separation and small betatron amplitude:

$$
\begin{aligned}
& \xi_{x}=-\frac{r_{e} N \beta_{x}}{2 \pi \gamma} \frac{1}{X^{2}}, \\
& \xi_{y}=\frac{r_{e} N \beta_{y}}{2 \pi \gamma} \frac{1}{X^{2}} .
\end{aligned}
$$

\section{HEAD-ON AND LONG RANGE BEAM- BEAM TUNE SPREAD}

The tune shift spread in a collider machine is one of the most important parameters in its design and operation. The spread must be limited to those values that avoid crossing the dangerous resonance in the operation tune space of the machine. This crossing may produce instabilities in the beam, limiting the performance of the machine (beam lifetime and luminosity) and creating radiation problems in the detectors (beam-halo). This tune shift spread is due to the nonlinear (in general) force felt by particles of the two interacting bunches.

The particle transverse motion can be written as

$$
X(s)=a_{x} \sigma_{x} \sin \left(\phi_{x}(s)+2 \pi Q_{x} k\right),
$$

where, $a_{x}$ is the transverse (horizontal and vertical) amplitude of the particle measured in units of the beam size, $k$ is the turn number, $Q_{x}$ is the operational tune of the machine, and $s$ represents the azimuthal position of the collision.

The particle position be unperturbed, it is easy to show that the angular kick gives the following phase displacement

$$
\Delta \phi_{x}=-\frac{\beta_{x}}{a_{x} \sigma_{x}} \Delta x^{\prime} \sin \varphi_{x},
$$

where $\varphi_{x}=\phi_{x}(s)+2 \pi k Q_{x}$. The phase displacement takes different values from one turn to another. Let's define the tune shift through the mean value of the phase fluctuation over many turns:

$$
\Delta Q_{z}=\frac{1}{2 \pi}\left\langle\Delta \phi_{z}\right\rangle
$$

It follows that

$$
\begin{gathered}
\frac{\Delta Q_{x}}{\xi_{x 0}}=\frac{\sigma_{x}\left(\sigma_{x}+\sigma_{y}\right)}{2 \pi^{2} a_{x}} \int_{0}^{2 \pi}\left(d_{x}+a_{x} \sin \varphi_{x}\right) \sin \varphi_{x} d \varphi_{x} \int_{0}^{2 \pi} d \varphi_{y} \\
\left.\int_{0}^{\infty} \frac{\exp \left[-\frac{\left(d_{x}+a_{x} \sin \varphi_{x}\right)^{2} \sigma_{x}^{2}}{2 \sigma_{x}^{2}+t}-\frac{\left(d_{y}+a_{y} \sin \varphi_{y}\right)^{2} \sigma_{y}^{2}}{2 \sigma_{y}^{2}+t} d t\right.}{\left(2 \sigma_{x}^{2}+t\right)^{3 / 2}\left(2 \sigma_{y}^{2}+t\right)^{1 / 2}} d 11\right) \\
\frac{\Delta Q_{y}}{\xi_{y 0}}=\frac{\sigma_{y}\left(\sigma_{x}+\sigma_{y}\right)^{2 \pi}}{2 \pi^{2} a_{y}} \int_{0}^{2 \pi}\left(d_{y}+a_{y} \sin \varphi_{y}\right) \sin \varphi_{y} d \varphi_{y} \int_{0}^{2 \pi} d \varphi_{y} \\
\int_{0}^{\infty} \frac{\exp \left[-\frac{\left(d_{x}+a_{x} \sin \varphi_{x}\right)^{2} \sigma_{x}^{2}}{2 \sigma_{x}^{2}+t}-\frac{\left(d_{y}+a_{y} \sin \varphi_{y}\right)^{2} \sigma_{y}^{2}}{2 \sigma_{y}^{2}+t}\right]}{\left(2 \sigma_{x}^{2}+t\right)^{3 / 2}\left(2 \sigma_{y}^{2}+t\right)^{1 / 2}} d t
\end{gathered}
$$

where $d_{x}=X / \sigma_{\mathrm{x}}$ and $d_{y}=Y / \sigma_{y}$ are the relative separation between the orbits of the colliding bunches.

Figure 5 and 6 show the relative horizontal and vertical head-on tune shift. The short-range tune shift decreases with increasing amplitude and it decreases faster in horizontal direction. Figure 7 and 8 give the long-range relative horizontal and vertical tune shift with $d x=8.0$. The long-range effect depends strongly on the beam separation and is strongest when the particle amplitude approaches 
the beam separation. In order to get small tune spread, the horizontal amplitude should be smaller than $4 \sigma_{x}$. The vertical amplitude has very small effect on the tune spread if the horizontal amplitude is smaller than $4 \sigma_{x}$. It means that there is potential advantage of vertical injection over horizontal injection when beams are separated in horizontal direction. Chin Yong Ho[3] got the same conclusion by simulation method. Horizontal injection will has more advantage when vertical injection is chosen. This is shown in figure 9 and 10.

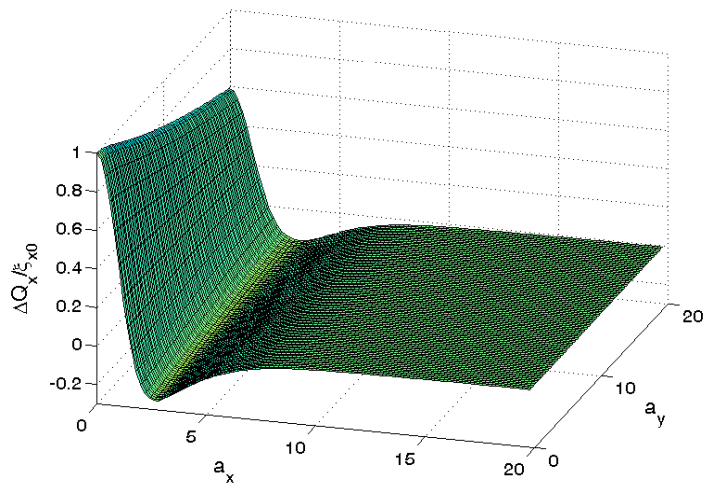

Figure 5 Relative horizontal head-on tune shift

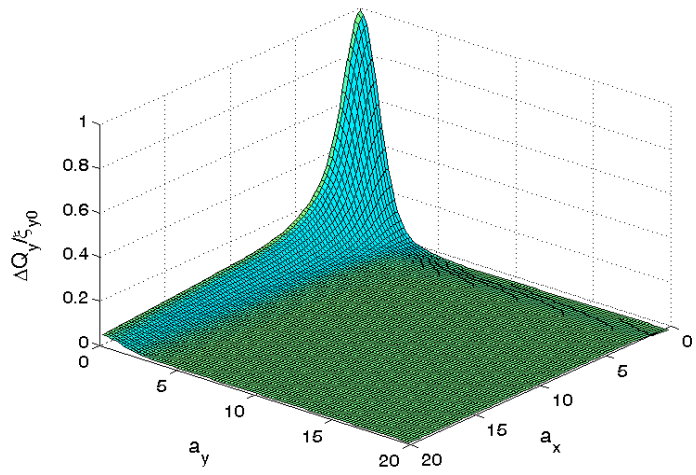

Figure 6 Relative vertical head-on tune shift

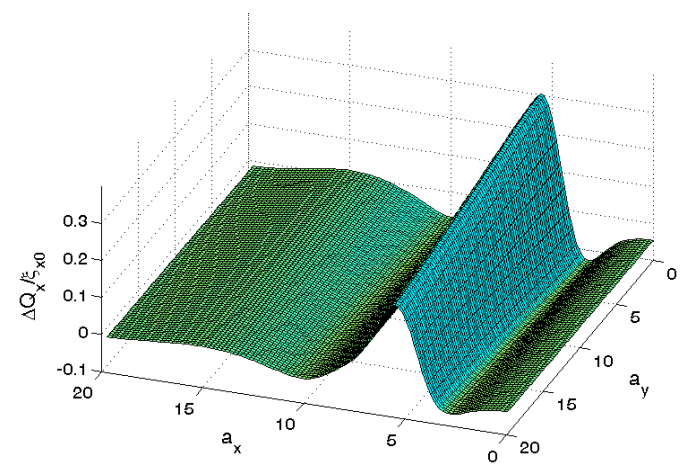

Figure 7 Relative horizontal tune shift with $d x=8.0$.

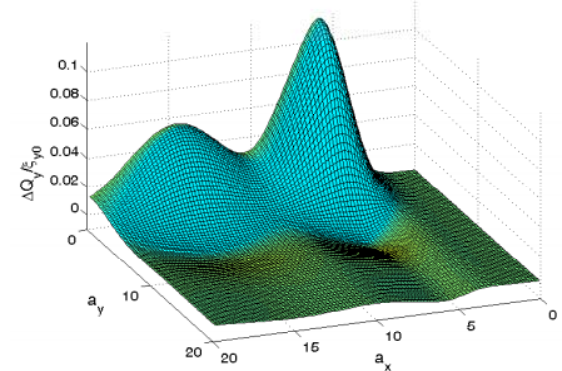

Figure 8 Relative vertical tune shift with $d x=8.0$.

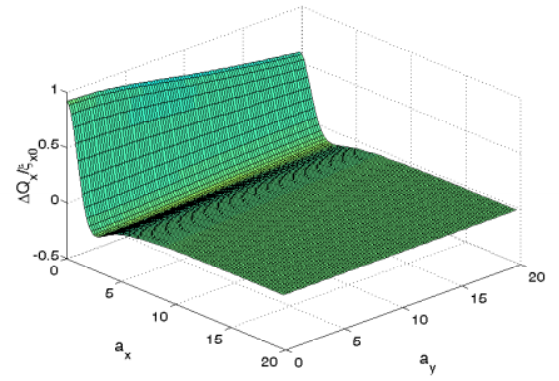

Figure 9 Relative horizontal tune shift with $d y=100.0$.

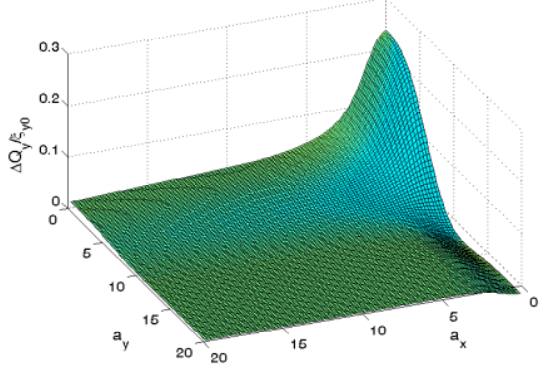

Figure 10 Relative vertical tune shift with $d y=100.0$.

\section{CONCLUSION}

The shift and spread from head-on and long-range beam-beam interactions for flat beam have been studied with analysis method. Resulting simple analytic formulas can be used to optimize the operation parameters. The analytic method is more simple and clear to understand than the simulation method.

\section{REFERENCE}

[1] W. Herr, Tune Shift and Spreads Due to Short and Long Range Beam-Beam Interaction in the LHC, CERN SL/90-06, 1990

[2] G. Lopez, Head-On and Long Range Beam-Beam Tune Shifts Spread in the SSC, SSCL-442, 1991

[3] Yong Ho Chin, Effects of parasitical Beam-Beam Interaction During the Injection Process, LBL-32468, 1992

[4] Kohji HIRATA, A Symplectic Beam-Beam Interaction with Energy Change, KEK Preprint 92117. 